\title{
PROFESORES EXCELENTES: EL FIN DE LA UTOPÍA EN EL DISCURSO
} PEDAGÓGICO CONTEMPORÁNEO

\author{
Great Teachers: The end of utopia in contemporary pedagogical discourse
}

\author{
GUIDO NICOLÁS ÁLVAREZ \\ Facultad de Ciencias Sociales, Universidad de Buenos Aires \\ Galvarez@sociales.uba.ar
}

\section{RESUMEN}

Desde la modernidad la utopía ha sido uno de los dispositivos fundamentales del discurso pedagógico. Sin embargo, en los tiempos actuales de educación como razón de lo diverso y de caídas de grandes relatos, el contenido utópico de los textos pedagógicos pareciera ser dejado a un lado para centrarse en específico en lo procedimental. En el presente artículo intento proponer una lectura del tratamiento ético del discurso pedagógico contemporáneo, tomando como representante al documento Profesores Excelentes (Bruns \& Luque, 2014), en la que el abandono de este tradicional dispositivo no es entendido como sinónimo de ausencia de contenido normativo, sino como reconfiguración del mismo en sintonía con su contexto sociopolítico, es decir, encuentro que esta redefinición negaría la utopía, pero no los compromisos ético-políticos.

Palabras clave: Calidad educativa; discurso pedagógico; sujeto pedagógico; utopía.

\section{ABSTRACT}

Utopianism has been one of the fundamental strategies in pedagogical discourse ever since the modern era. However, in current times which featured by the loss of great story tales, the utopian content in pedagogy seems to be left aside to focus mainly on instructional terms. In the present article, I encourage the reading of the ethical content of "Great Teachers" (Bruns \& Luque, 2014), considered a reflection of contemporary pedagogy, where the absence of this traditional strategy is not seen as a synonym of lack of normative content, but a reconfiguration of it, in the same frequency as its socio-political context. In other words, this redefinition may indeed reject utopia, yet not its ethical-political commitments.

Key words: Educational quality; pedagogical speech; pedagogical subject; utopia.

\section{INTRODUCCIÓN}

Podría considerarse que desde los orígenes de la escuela sus promotores, los pedagogos, han sabido muy bien que el emplazamiento de una institución de educación 
sistematizada, simultánea, mutua y monopólica del saber y de las costumbres requiere dar razones que respondan al por qué los niños han de asistir a ella y no más bien recibir instrucción familiar asociada a las necesidades específicas de cada hogar, gremio o pequeña comunidad. Tal institución generaría tensiones con tradiciones y conocimientos particulares, por lo que su justificación debería extenderse al campo de lo ético, además de referirse a los progresos posibles por la alfabetización e instrucción en saberes formales. Sabían, en definitiva, que la escuela debería de ser vindicada, defendida y legitimada para ser justificada.

Y así lo hizo Comenius (1986), hito del discurso pedagógico en la modernidad, quien respecto al posible cuestionamiento por la conveniencia de la educación escolarizada respondió en su Didáctica Magna que la formación ordenada deviene en necesaria si el hombre ha de ser tal (p. 55). Y por "tal" entendía un ser de "ciencia y virtud" (p. 59), debiendo regir como norte "para todos sin distinción"(p. 60) desde reyes a súbditos. De esta manera, y siguiendo la hipótesis de Narodowski (1994) en la génesis de la actual reflexión en relación a lo escolar aparecía postulado un deber ser antropológico en que la escolarización se justificaría insertándose como medio para un fin que efectivamente trazaba un nuevo horizonte que ponía en tensión el orden de su época para un siglo XVII aún aristocrático ${ }^{1}$ (Hamilton, 1993). Un relato que presentaba una humanidad deseada no realizada en el presente, un "punto de fuga" que ordena el curso hacia el destino final (Narodowski, 1994); una utopía (Mannheim, 2004).

Tras Comenius (1986), los subsiguientes grandes pedagogos incluyeron este carácter utópico en sus reflexiones en torno a lo educativo, extendiéndose la lista hasta bien entrado el siglo XX con las obras de P. Freire, J. Dewey y finalmente Iván Illich (Narodowski, 1999); Sin embargo, algo ha cambiado. El discurso pedagógico contemporáneo parecería haberse desprovisto de tal dispositivo. Frente a su carácter universal, los faros sociopolíticos de la pedagogía contemporáneas claman por la comprensión de lo singular, y frente a su carácter disciplinante, exaltan la tolerancia (Narodowski, 1999). Caen los grandes relatos (Lyotard, 1987). De hecho, en algunos casos la moderación respecto de los compromisos normativos es tal que países con regímenes de gobierno no democráticos llegan a considerarse modelos de excelencia educativos en trabajos como los de Forestier, et al. (2016) o Gopinathan \& Lee (2018), entre otros. Narodowski observa y resume muy bien este movimiento sosteniendo que actualmente la pedagogía "parece plegarse a la búsqueda de un modelo perfecto de enseñanza, un modelo sin fisuras que permita procesar adecuadamente y sin errores la transmisión de conocimientos" (1999 p. 30). Así, el discurso educativo actualmente se

\footnotetext{
${ }^{1}$ Se considera que solo después de la Revolución Francesa se consigue el pasaje de una sociedad organizada en castas bajo el principio de igualdad formal (Hobsbawm, 1996).
}

34 | INTEREDU № 3 VOL. II (DiCIEMBRE 2020) PÁGs. 33-42. ISSN: 2735-6523 
inscribiría en el fenómeno del fin de la utopía entendido como caída de relatos cuyos anhelos trascienden disruptivamente la situación social (Mannheim, 2004)² lo que daría pie a una serie de trabajos pedagógicos cuyas líneas estarían dedicadas a la reflexión didáctica.

Sin embargo, esta situación sería contradictoria con la exigencia de dar cuenta del sentido por el que educar, descrita arriba. En este sentido podría ensayarse que frente al fin de la utopía ocurra una reconfiguración del contenido normativo que justifica la escolarización, antes que una negación del mismo. De manera que podrían encontrarse valoraciones éticas, pero no como puntos de fuga a un nuevo y distinto destino, sino como faros éticos que guían nuestro propio rumbo. Esto es compromisos políticos, explícitos o no, con el presente antes que un nimio vacío de sentido.

En este marco de preocupaciones surge este breve artículo en que intento extender la hipótesis precedente al estudio del documento Profesores Excelentes: Cómo Mejorar el Aprendizaje en América Latina y el Caribe (Bruns, et al., 2014) evaluando si acaso puede subsumirse como ejemplo de la misma. Ciertamente, sería fácil corroborar la tendencia propuesta dado que, según reza su subtítulo, el trabajo está dedicado a revisar los aspectos metodológicos de la enseñanza. Sin embargo, también resulta importante rastrear sus elementos normativos y evaluar su cualidad con el objetivo de elucidar los fines allí postulados para legitimar el proceso de escolarización (Avanzini, 1994).

En particular, Profesores Excelentes explicita sus compromisos postulando como norte ético-político de la enseñanza, así como promover la "capacidad para resolver problemas, la disposición al aprendizaje continuo y el pensamiento crítico" (2014, p. 1). Capacidades distintivas de lo que será el "capital humano" que habrá de "promover las condiciones para la innovación y la mejora de la productividad en la región" (Bruns, et al., 2014, p. xi). A partir de allí, al contrastarlos con los atributos de un discurso utópico, de acuerdo con la clásica conceptualización de Mannheim en Ideología y Utopía (2004), encuentro que las valoraciones normativas presentes en la publicación del Banco Mundial no pueden ser consideradas como utopías en tanto que no "resultan incongruentes con el estado real dentro del cual ocurren" (Bruns, et al., 2014, p. xi), reafirmando así la hipótesis de Narodowski (1999). La praxis escolar es justificada en sintonía con el propio presente. Una época signada, entre otras cosas, por la expansión de las relaciones sociales de producción capitalistas a nivel global y de confianza en la iniciativa individual para la innovación y el desarrollo por sobre

\footnotetext{
${ }^{2}$ Hay otras conceptualizaciones posibles acerca de la naturaleza del “fin de las utopías” y sus implicancias. Entre ellas, es muy famoso el trabajo de Marcuse de título homónimo, al respecto puede ser interesante consultar Donskis, L. (1996). En este trabajo cuando me refiero al fin de la utopía me refiero a la caída de un tipo de rela to normativo con los atributos que le asigna Mannheim (2004).

Por otro lado, hay estudios actuales que revisan el éxito teórico de la hipótesis del fin de las utopías., por ejemplo: Sargent (2005). Aunque por la brevedad del trabajo no puedo más que mencionarlo.
}

INTEREDU № 3 Vol. II (Diciembre 2020) PÁGS. 33-42. ISSN: 2735-6523| 35 
la propia capacidad estatal (Held, 1997; Harvey, 1989) ${ }^{3}$. Sin duda tal proyecto educativo es congruente con su situación (Brown \& Lauder, 1996).

Por último, la selección del documento como relevante y representante del discurso pedagógico contemporáneo responde a que considero que puede inscribirse en lo que Avis et al. identifican como el new consensus (1996), esto es la primacía de variables económicas para la orientación de las políticas educativas (Ball, 1998). Asimismo es destacable su carácter reciente, su enorme base empírica ${ }^{4}$ y su publicidad producto de haber sido patrocinado por el Grupo Banco Mundial. De esto último le sigue haber sido citado en más de un centenar de estudios de educación en la región y además, haber sido referido en distintos proyectos de políticas públicas, por ejemplo el Plan Maestr@en Argentina ${ }^{5}$. Por esta relevancia, un trabajo con intereses descriptivos del contenido normativo de este documento puede iluminar aspectos interesantes respecto a la nodal pregunta por el tipo de subjetividad que nos proponemos coadyuvar a formar en nuestra práctica docente (Cohan, 1996).

\section{PROFESORES EXCELENTES: DE UTOPÍAS A MEJORAS}

Evaluar cómo se organiza y cuál es la naturaleza del contenido normativo de un texto, en este caso Profesores Excelentes, a fin de contrastarlo con el concepto de "utopía", puede considerarse que supone una doble complejidad. No sólo requiere una lectura transversal del mismo, teniendo en cuenta que el grueso de su desarrollo está dedicado a aportar evidencia para informar una futura política pública, sino que a su vez el propio término mentado está atravesado por obstáculos que hacen difícil su conceptualización. De acuerdo con el estudio de Ricœur (2008) podrían señalarse cuatro dificultades para considerarlo como un problema autónomo ${ }^{6}$. La primera de ellas es que el término tiende a ser reconocido como un género literario (p. 289). La segunda se manifiesta en la actitud en la que la abordamos dado que "suscita una especie de complicidad o connivencia por parte del lector bien dispuesto" (p. 290). Luego "el hecho de que las utopías (en plural) no expresan

\footnotetext{
${ }^{3}$ Claro que esto no es una descripción unívoca ni monocolor. Por un lado, hay diversos grados de desarrollo capitalista con diversas formas de participación del Estado y por otro, hay voces disidentes a esa confianza en la iniciativa privada. Sin embargo, la descripción sirve como gran trazado para observar tendencias generales. Así, al aumentar la denotación se reduce la connotación.

${ }^{4}$ Para el trabajo se observaron más de 15.000 clases en más de 3.000 escuelas de 7 países distintos, por lo que este trabajo constituye el estudio internacional de mayor escala de este tipo que se haya realizado.

5 Ver proyecto de Ley Plan Maestr@ (2017). Disponible en: http://www.bnm.me.gov.ar/giga1/documentos/EL005527.pdf [fecha de consulta: 02/06/2020].

${ }^{6}$ De acuerdo con el autor estas situaciones también constituyen un obstáculo epistemológico para observar a la utopía en paralelo y "relacionadas con la ideología" (Mannheim. 2004, p. 289). Para adecuar la presentación a los fines de este trabajo se hace énfasis en lo referente a la conceptualización.
}

36 | INTEREDU № 3 VOL. II (DiCIEMBRE 2020) PÁGs. 33-42. ISSN: 2735-6523 
fácilmente una significación central de la utopía (en singular)" (...) situándonos "frente a una pluralidad de utopías que resultan muy difícil de reunir con su nombre" (290). Y finalmente que el pensamiento marxista, el cual ha abordado el tema, "tiende a reducir las utopías a una subclase de ideología" de manera que devienen en "meros 'ecos', 'reflejos'” (291). Para salvar estos obstáculos, el autor se refiere a la obra de Mannheim (2004) a quien le reconoce la virtud teórica de precisamente haber "suministrado un concepto [de 'utopía'], una hipótesis operante de su indagación" (p. 292); y aquí se considera lo mismo.

En el ya clásico Ideología y Utopía de Mannheim (2004), "utopía" es definido como un "estado de espíritu (...) [que] resulta incongruente con el estado real dentro del cual ocurre" (p. 229) pero que logra transformar la realidad histórica. La incongruencia se produce porque esta idea "se orienta hacia objetos que no existen en una situación real" (...) "se 'aparta de la realidad' y tiende a "destruir, ya sea parcial o completamente, el orden de las cosas existente en determinada época" (Mannheim, 2004, p. 229). Una utopía trasciende la sociedad rompiendo los lazos del orden prevalente en su momento. El propio autor propone por ejemplo el postulado de la libertad de la burguesía ascendente previo a los acontecimientos de 1789 como discurso utópico. Y en torno a este destino deseado se “ordenan las conductas hacia elementos que no contiene la situación” logrando con esta "contraactividad" modificar "la realidad histórica existente en algo que esté más de acuerdo con sus propias concepciones" (p. 233). Esta es la utilización específica del concepto de "utopía" que sigo en el trabajo y que se refiere a algo distinto que a meras esperanzas éticas. Ciertamente hay otros atributos de la definición que ofrece el autor. Siguiendo la presentación de Ricœur (2008) "estar sustentada por grupos que se hallan en vías de ascenso" (p. 292), ser "un deseo dominante", "constituir una mentalidad, un Geist (..) un sistema simbólico general" (p. 293), "ser antagónica respecto de las demás" (p. 294), y “estar en vías de ser realizado" (p. 295). También Mannheim añade la dimensión temporal a su análisis dado que sostiene que una utopía es aquella que parece irrealizable sólo desde el punto de vista de un orden vigente" (2004, p. 233); Sin embargo, a los fines del trabajo sirve aquel criterio de demarcación según el cual si el contenido normativo no se estructura trascendiendo la realidad en que es producido, entonces no podemos decir que estamos frente a una utopía.

A la luz de esta conceptualización el rastreo de los preceptos normativos de Profesores Excelentes (2014) permitiría seguir que estos no se estructuran bajo el registro de la utopía dado que son congruentes con el propio presente. En efecto, desde el comienzo hasta el final del documento aparece la impronta de potenciar las condiciones de vida actuales. En sus propias palabras:

Se espera que este libro, que reúne en un solo volumen las principales reformas de políticas docentes que están en marcha actualmente en la región y las mejores 
evidencias disponibles sobre su impacto, sirva para estimular y apoyar el progreso acelerado que se requiere (p. 51).

Como se observa, fomentar el desarrollo de las economías de la región aparece explícitamente sostenido como fin. Aunque para encontrarlo no es necesario esperar al último párrafo, al contrario, puede observarse ponderado en cada una de las líneas argumentales. Como intentaré mostrar de forma sintética, las grandes hipótesis del documento establecen una relación entre una recomendación para mejorar la enseñanza como variable antecedente, y la "calidad" y/o "eficiencia" como su consecuente. Y esto último se asocia por evidencia cuantitativa al aumento de la calidad de vida de cada país, que opera como meta anhelada.

El documento parte de una relación muy clara: “un país cuyo desempeño promedio en las pruebas internacionales es una desviación estándar superior a otro (aproximadamente 100 puntos en PISA) obtendrá cerca de 2 puntos porcentuales más de crecimiento anual del PIB a largo plazo" (Bruns et al., 2014, p. 3). Aquí entonces el compromiso que guía la producción del informe. Y a esto le agrega que la calidad de la educación -entendida como la variación en los aprendizajes de contenidos evaluados en las pruebas internacionales- está condicionada por la calidad de los profesores (p. xi) ${ }^{7}$.

Con tal argumento, se apoya en otros estudios para medir la "calidad educativa", específicamente PISA, y la "calidad de vida", por variación en el PIB tomando como fuente al BM, y a partir de allí avanza en una serie de recomendaciones, que sirven también como indicadores, para mejorar la enseñanza. Estas propuestas se resumen en tres esferas prioritarias para las reformas de políticas educativas: "reclutar mejores profesores" (pp. 2334), “desarrollar profesores de excelencia" (pp. 34 -40) y finalmente "motivar a los profesores para que mejoren su desempeño" (pp. 40-50). A las cuales le dedica un capítulo a cada una del 3 al 5, respectivamente.

Aquellas propuestas se inscriben más adecuadamente dentro de la disciplina de las políticas educativas, por lo que me centraré en sus dos capítulos precedentes en que los autores ciñen la mirada a aspectos estrictamente pedagógicos de la enseñanza y ofrecen respuestas a para qué educar. En el capítulo 1 se evalúa “la importancia profesores" (pp.311) en el aprendizaje y en términos sociales en general, y en el capítulo 2 se presenta un informe cuantitativo en torno a qué hacen "los profesores latinoamericanos en el aula" (pp.11-23).

\footnotetext{
${ }^{7}$ A modo esquemático podría pensarse que el argumento es un silogismo deductivo con variación proporcional positiva entre las tres variables, en el que la tercera cumple el lugar del fin: Calidad de Profesores $\propto$ Calidad Educativa; Calidad Educativa $\propto$ Calidad de Vida .: Calidad de Profesores $\propto$ Calidad de Vida.

38 | INTEREDU № 3 VOL. II (DiCIEMBRE 2020) PÁGs. 33-42. ISSN: 2735-6523
} 
Respecto de primer punto el documento es claro desde el comienzo. La importancia de los profesores está dada por su gran impacto económico (p. 1). Son los actores principales (p.5) en producir los beneficios económicos de invertir en educación teniendo en cuenta que "las diferencias entre el nivel promedio de las capacidades cognitivas de los países se correlacionan sistemática y sólidamente con las tasas de crecimiento económico a largo plazo" (p.3). En este sentido, la calidad del docente condiciona la calidad aprendizaje reducida a saberes capaces de ser evaluados en pruebas estandarizadas- lo que repercutirá en la tasa de crecimiento económico. El diferencial de la enseñanza deviene entonces en impulsar el aprendizaje, por lo que su actividad puede medirse como "valor agregado" a su producto entendido como la cantidad de saberes aprendidos por el alumno (p. 6).

Lo mismo ocurre con el segundo aspecto. Al evaluar qué hacen los docentes en el aula, los autores presentan un estudio de acuerdo a la "eficacia" del tiempo de clase, tomando en consideración que "la intensidad con que se aprovecha este momento de instrucción es un determinante clave de la productividad del gasto en educación" (p.11). Bajo esta consideración encuentran que el tiempo dedicado estrictamente a la instrucción es bajo en comparación con otras regiones (p.12) y que se recurre poco a materiales didácticos asociados a las nuevas tecnologías (p.15) lo que influye en que no se capte la atención de todos los alumnos (p.17). Por lo que se concluye que en general hay dificultades en torno al estar "preparados para usar dichas estrategias y cada minuto de la hora de clase con eficacia" (p.18).

Finalmente, tras haber explicitado su compromiso con una educación para el capital humano, el documento construye indicadores para "reclutar, desarrollar y motivar a una nueva generación de profesores" (p.50) que se justifican entonces por su carácter de antecedente para fomentar mejores niveles de aprendizajes cuantificables en pruebas estandarizadas en vistas de potenciar el desarrollo de la región. Como es manifiesto, el fin que se persigue en esta publicación tiene la forma de compromiso político con el "desarrollo" antes que de ordenamiento de las conductas hacia elementos que no contiene nuestra situación sociopolítica (Mannheim, 2004). Tal como se sostiene en el último párrafo, se propone una mejora de las condiciones actuales, entendida como aumento de la riqueza en economías capitalistas, antes que una utopía.

\section{REFLEXIONES FINALES}

He partido de la hipótesis de Narodowski (1994; 1999) según la cual desde Comenius (1986) la utopía ha sido uno de los dispositivos fundamentales del discurso pedagógico, pero se observa que en los tiempos actuales de caídas de grandes relatos (Lyotard, 1987), el contenido utópico de los textos pedagógicos pareciera ser dejado a un lado para centrarse específicamente en lo procedimental. Y bajo esta consideración puede leerse el tratamiento 
de los compromisos éticos de Profesores Excelentes teniendo en cuenta que persigue fines congruentes con su actualidad. En efecto puede observarse que el abandono de este tradicional dispositivo no es sinónimo de ausencia de contenido normativo, sino reconfiguración del mismo en sintonía con su contexto sociopolítico asumiendo y explicitando compromisos ético-políticos con la eficiencia del sistema educativo para el mercado laboral. No postula nortes disruptivos sino que manifiesta el compromiso político de formar el "capital humano" capaz de promover los indicadores económicos de la región. Lo cual se inserta en la obra como fin de la reforma educativa.

Pero esto último no se encuentra desprovisto de tensiones. Por un lado, la obra es recorrida por una concepción instrumental que reduce al tiempo en el aula a mera variable para la eficiencia en una relación costo-beneficio. Aunque podría pensarse que el tiempo es también condición para el encuentro, la socialización y el desarrollo de vínculos de confianza y respeto necesarias para la dinámica educativa. ¿Acaso podría ir tal concepción en detrimento de lo segundo? Por otro lado, se presenta en el documento una visión mecánica de la enseñanza como transmisión de saberes desprovista de la evaluación de su comunidad de sentido. El supuesto de la neutralidad del educar es tal que llega a afirmarse: "puede parecer obvio que un año de educación en Malí no sea equivalente a uno en Singapur" (Bruns \& Luque, 2014, p. 3). Lo que convalida la posibilidad de que un país declaradamente autocrático eduque bien. Esto, por citar tan sólo dos elementos que considero pasibles de revisión. Por ello, sería una reducción considerar que este fin en particular es la única forma de compromiso con la condición actual frente al distanciamiento de lo utópico.

En este sentido cabe la preocupación respecto de la valoración de lo que podría asumirse como la caída de las utopías. En este punto y siguiendo la lectura de Ricœur (2008) el propio Mannheim observa con cierta ambivalencia este fenómeno. En el apartado La Utopía en la Situación Contemporánea (2008) al tiempo que reconoce cierta positividad en tanto que esto implicaría una "aproximación a la vida real", un "dominio de las condiciones concretas de la existencia" (p. 285), también observa cierta "esterilidad" (Ricœur, 2008) en tanto se desintegran las visiones generales del mundo. En una lectura similar, Sargent sostiene que en el estudio de Mannheim, mientras que tanto las ideologías como las utopías postulan problemas, las segundas deben ser mantenidas ya que incluyen las semillas necesarias para el cambio social ${ }^{8}$ (2008, p. 267).

De manera que, frente a la divergencia en sentidos y futuros habilitados en el vacío de axiologías que dejan las utopías, podrían recuperarse otras miradas que asignen valoraciones distintas a la pregunta por el sentido del educar y sitúen su compromiso

\footnotetext{
${ }^{8}$ La traducción es propia.

40 | INTEREDU № 3 VOL. II (DicieMBRe 2020) PÁGs. 33-42. ISSN: 2735-6523
} 
político en dimensiones del presente no contempladas por el norte que orienta a la formación como capital humano. En tanto que la condición actual no se reduce únicamente a la expansión de las relaciones de mercado, el fin de la utopía puede dar lugar a otras vindicaciones que emplacen en primer lugar la bandera de la democracia, el pluralismo, la diversidad o el reconocimiento, por nombrar algunos ejemplos de dimensiones también actuales.

Y aquí Adorno (1998) puede ofrecernos algo de ayuda tan solo en clave propositiva respecto a otras posibilidades a la interrogación respecto del pro-yecto sin utopías. El 18 de abril de 1966, día en que se cumplía el $20^{\circ}$ aniversario de la creación de las Naciones Unidas y del Tribunal Internacional de Justicia que juzgaría las atrocidades cometidas durante la Segunda Guerra Mundial, el pensador alemán brindó una conferencia radial con motivo a la educación. Ya anciano y probablemente pensando en las próximas generaciones, decidió abrir su conferencia sin miramientos por presentaciones ni introducciones. Tan pronto se encendió el micrófono, nuestro autor pronunció: “la más urgente demanda que recae sobre toda educación es que Auschwitz no se repita" (Adorno, 1998, p. 1) ${ }^{9}$. Aunque habían transcurrido algo más de dos décadas desde el fin a tal horror, a Adorno le preocupaba observar cierta tendencia enraizada en lo humano a la barbarie, un peligroso movimiento anti-civilización que podría estallar en cualquier momento. Frente a ello solicitaba a la comunidad docente aunar esfuerzos en torno a una educación centrada específicamente en la autodeterminación (Adorno, 1998, p. 3), o sea en el imperativo kantiano del pensar por sí mismo (p. 4). De ello derivaba otros atributos de esta formación como ser no colectivista (p. 5), no nacionalista - en su acepción totalitaria- (p. 10), opuesta a la virilidad asociada a la "dureza" y a la violencia sobre el cuerpo (p. 5) y de racionalidad objetiva, i.e. que no fetichice los fines (p. 7). En fin, una educación para el amor (Adorno, 1998, p. 7).

La propuesta de Adorno respecto del sentido del educar asociado centralmente a la formación de subjetividades capaces de reconocer y someter a reflexión crítica su propia "frialdad" para con otros (p. 8), se presenta al ojo contemporáneo en tensión con el faro propuesto en Profesores Excelentes para la reforma educativa. Nos propone otro norte posible caracterizado por la formación humana como ser autónomo antes que como capital. Centra su interés no tanto en la promoción de la economía sino en la prevención del autoritarismo. Mira el presente con interés en los procesos democráticos más que en la internacionalización de las relaciones de mercado. De esta manera, educar para no repetir Auschwitz es un compromiso congruente con un tiempo también caracterizado por luchas por el reconocimiento (Fraser, 1995) y de apuesta por el respeto a la singularidad (Foucault, 1999)

El fin de la utopía establece invariablemente los compromisos políticos en el presente. Lo que sí puede variar es en qué elemento de la actualidad fijar la atención. Y esto es un

\footnotetext{
${ }^{9}$ La traducción es propia.
} 
punto nodal a tener en cuenta en el estudio de Profesores Excelentes. Reconociendo que la escolarización tiene efectos subjetivantes, resultaría poco cauteloso pormenorizar el tratamiento de la pregunta por el para qué educar y con qué anhelos en vistas a la toma de decisión en políticas públicas y las prácticas y teorías educativas en general.

\section{BIBLIOGRAFÍA}

Adorno, T. W. (1998). Education after Auschwitz. Critical models: Interventions and catchwords, 191, 204.

Avanzini, G. (1994). Les trois fonctions de la philosophie de l'éducation. Pour une philosophie de l'éducation. Dijon: CNDP-CRDP de Bourgogne.

Avis, J., Bloomer, M., Esland, G., Glesson, D. \& amp; Hodkinson, P. (1996). Knowledge and Nationhood: education, politics and work (London, Cassell).

Ball, S. J. (1998). Big policies/small world: An introduction to international perspectives in education policy. Comparative education, 34(2), 119-130.

Brown, P. \& Lauder, H. (1996). Education, globalisation and economic development, Journal of Education Policy, 11, pp. $1 \pm 25$.

Bruns, B., Luque, J., De Gregorio, S., Evans, D., Fernández, M., Moreno, M., ... \& Yarrow, N. (2014). Profesores excelentes: cómo mejorar el aprendizaje en América Latina y el Caribe. Banco Mundial.

Cohan, W. (1996). Filosofía de la educación. Algunas perspectivas actuales. Salamanca: Ediciones Universidad de Salamanca.

Comenius, J. A. (1986). Didáctica magna. Madrid: Ediciones Akal.

Donskis, L. (1996). THE END OF UTOPIA? Soundings: An Interdisciplinary Journal, 79(1/2), 197-219. Retrieved June 8, 2020, from www.jstor.org/stable/41178748

Forestier, K., Adamson, B., Han, C., \& Morris, P. (2016). Referencing and borrowing from other systems: The Hong Kong education reforms. Educational Research, 58, 149-165. doi:10.1080/00131881.2016.1165411

Foucault, M. (1999). ¿Es inútil sublevarse?. Estética, ética y hermenéutica. Obras esenciales, 3, 2037. Barcelona: Ed. Paidós.

Fraser, N. (1995). From redistribution to recognition? Dilemmas of justice in a'postsocialist'age. New left review, 68-68.

Gopinathan, S., \& Lee, M. H. (2018). Excellence and equity in high-performing education systems: policy lessons from Singapore and Hong Kong/Excelencia y equidad en sistemas educativos de alto rendimiento: lecciones de las políticas educativas en Singapur y Hong Kong. Infancia y Aprendizaje, 41(2), 203-247.

42 | INTEREDU № 3 VOL. II (DicIEMBRE 2020) PÁGS. 33-42. ISSN: 2735-6523 
Hamilton, D. (1993). Comênio e a nova ordem. Revista Pro-Posições, 4(2).

Harvey, D. (1989). The Condition of Postmodernity. Oxford: Blackwell.

Held, D (1997). La Democracia y el orden global. Barcelon: Paidos.

Hobsbawm, E. (1996). Age of revolution: 1789-1848. New York: Vintage Books.

Lyotard, J. F. (1987). La condición posmoderna: informe sobre el saber. Madrid: Ediciones Cátedra S.A.

Mannheim, K. (2004). Ideología y utopía: introducción a la sociología del conocimiento. Ciudad de México: Fondo de cultura económica.

Narodowski, M. (1994). Infancia y poder. Buenos Aires: Aique

— (1999). Después de clase: desencantos y desafíos de la escuela actual. Buenos Aires: Noveduc Libros.

Narodowski, M., \& Botta, M. (2017). La mayor disrupción posible en la historia de la pedagogía moderna: Ivan Illich. Pedagogía y saberes, (46), 45-54.

Ricoeur, P. (2008). Ideología y utopía. Barcelona: Gedisa. No se encuentra en el texto

Sargent, L. T. (2005). The necessity of utopian thinking: A cross-national perspective. Thinking utopia: Steps into other worlds, 4, 1.

Sargent, L. T. (2008) Ideology and utopia: Karl Mannheim and Paul Ricoeur, Journal of Political Ideologies, 13:3, 263-273, DOI: 10.1080/13569310802374479 